\title{
Simulation Experimental Study on Flow and Block of the Mixed Flow of Water and Sand Through the Broken Zone of Rocks
}

\author{
Delong Liu ${ }^{1, a}$, Chen Chen ${ }^{2, b}$, Wanghua Sui ${ }^{3, c}$ \\ ${ }^{1}$ Engineering College, Jilin University,Changchun, China \\ ${ }^{2}$ Engineering College, Jilin University, Changchun,China \\ ${ }^{3}$ School of Resources and Geosciences, China University of Mining and Technology, Xuzhou, \\ China \\ ae-mail: liudelong@foxmail.com, ${ }^{\text {be-mail: chenchen@jlu.edu.cn, }},{ }^{\mathrm{c}} \mathrm{e}-\mathrm{mailsuiwanghua@cumt.edu.cn}$
}

Key words: mixed flow of water and sand; caving rocks; quicksand; block

\begin{abstract}
A set of interior simulation experiments for quicksand were designed, which were carried out to study the law of flow and block for sand with different particle sizes and different water content from loose level which flow through the broken rocks above coal seam.The relation was obtained ,which was between speed ,total amount of quicksand and particle size of sand, water content of sand,channel size of broken rocks. The displacement time curve of sand column was fitted into linear function, polynomial function or exponential function. A mechanical model was established to analyze the transport mechanism of sand with different conditions flowing through the broken rocks. And the factors that affect the flow and block of sand were analyzed.Experiment results shows: when dry sand flows through the broken rocks, it is uniform and do not form block generally; wet sand almost all form block; all the mixed flow of water and sand occurs inrush of sand , and when the water level get lower than sand surface, it becomes wet sand and the inrush of sand get finished.In broken rocks, it is a prerequisite for the occurrence of quicksand that the water level in the aquifer is higher than the surface of sand. However, with such a condition of quicksand, the key factor on the speed and the amount of quicksand is the channel size. The study indicates that the establishment of mechanical model for macro analysis can explain the migration mechanism of the mixed flow of water and sand through the broken rocks.
\end{abstract}

\section{Introduction}

Inrush of sand is a mine geological disaster, which is thatthe mixed flow of water and sand with high sand content bursts into the underground working face passing through the overlying rock on the roof of coal seam and causes property losses and casualties while mining near loose layer .Along with extending the upper extraction limit in recent years, due to the thin overlying bedrock,mining-induced fractures caused by underground mining or the natural fractures zone are often connected with the overlying loose aquifer so that the phreatic water of the formation bursts into the underground coal face along fractures. When the width of fractures reaches a certain extent,it will often lead to inrush of sand and water in the coal face so that the working face will be buried and cause huge economic losses and casualties.

The predecessors have done a lot of research about water and sand bursting. Sui Wanghua, Yang Weifeng etc. ${ }^{[1,2,3]}$ researched the migration characteristics of the mixed flow of water and sand trough mining-induced fractures in the thin bedrock.They pointed out the cause of formation of channels which water and sand burst out from mining under thin bed rock and thick loose layer,and they also pointed out the relation between clay content and inrush of water and sand. Wu Yongping etc. ${ }^{44}$ researched the forces on sand granule before inrush based on laws of sediment incipient motion by giving out the mechanics model of sand pseudo structure,and gave out the theoretical discriminant occurred conditions of sand inrush based on the height of the aquifer. Lu Mingshi ${ }^{[5]}$ researched the mechanism of water and sand bursting in shallow buriedstope, and pointed out that the aquifer height is a key factor influencing water and sand pour. Liang Yankun ${ }^{[6]}$ researched the mechanism of sand inrush in the single fracture by introducing the theory of particle flow and established the discriminant of sand inrush,and so on. 
It shows that the previous studies on the flow of water mixed sand through the single fracture have been quite mature, but the research on the flow through the porous broken rocks is less.Therefore,in this paper, flow and block of the mixed flow of water and sand (including caving zoneand the natural fracture zone) would be studied. In order to be easy to study, according to the principle of similarity, the rock masses in the broken rocks were generalizedas spherical particles so that the irregular broken rocks were generalized as a kind of porous medium composed of spherical particles. A set of interior simulation experiments for quicksand were designed.By monitoring the falling velocity of the sand column and the water column and the residual volume of sand column, the law and the characteristics of the quicksand with different water content and different particle would be studied while passing through broken rocks.

\section{Experimental purposes, device and sample}

Experimental purposes.Because in engineering practice, the water and sand inrush is sudden and catastrophic, the characteristics and influence factors of the mixed flow of water and sand can't be studied by field test. In order to reproduce the phenomenon that the mixed flow of water and sand passes through fractured rock mass in engineering geology, a set of simulation experiments are designed according to the similarity criterion to study the flow law and block characteristics of which when sand with different particle sizes and different water content passes through broken rocks. The specific purpose is to obtain: the relation between the velocity of sand inrush and sand grain size, water content,channel size in broken rocks; the relation between the residual amount of sand afterinrush of sand and sand grain size, water content, channel size in broken rocks etc..

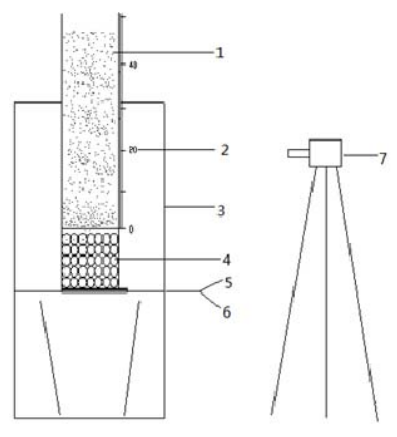

1-transparent long barrel filled with sand 2-ruler 3-support 4-simulate broken rocks 5-soft plastic sheet 6-wire mesh 7-camera

Figure 1.The schematic diagram of the device

Experimental device.Self-designed experimental device for sand inrush was used. Figure 1 shown is the schematic diagram of the device. The main parameters: (1) the glass bead size are: $25 \mathrm{~mm}, 21 \mathrm{~mm}, 16 \mathrm{~mm}$, respectively, marked for the No. 1, No. 2, No. 3 beads; (2) the wire of the mesh is about $0.6 \mathrm{~mm}$ in diameter and grids are all squares with the length of $7 \mathrm{~mm}$; (3) the long barrel is $1 \mathrm{~m}$ in height and $12 \mathrm{~cm}$ in diameter, and the short barrel is $6.6 \mathrm{~cm}$ in height.

Experimental sample preparation.In order to study sand with different particle size, prevent the interference of clay and other factors, and be able to shoot the experimental phenomenon more clearly, sand was strictly screened, repeatedly washed and dried. Here was a selection of sand which was 0.5-1.0mm, $0.25-0.5 \mathrm{~mm}, 0.075-0.25 \mathrm{~mm}$ in particle size, respectively, i.e. coarse sand, medium sand, fine sand, and was marked as No. 1, No. 2, No. 3 sand.

\section{Experimental Process}

According to the initial design and experimental progress, the experiments was divided by the different water content of sand into three stages, i.e. dry sand experiments, wet sand experiments and mixed flow of water and sand experiments.As we all know, dry sand can be considered that it does not contain water. However, the wet sand here was defined that it contained water before the saturation or near the saturation; and the mixed flow of water and sand was definedthat it was saturated with 
moisture and the water level was higher than the column of the sand.Steps are as follows :

i)When the device was ready, the sand waspoured into the long barrel to $40 \mathrm{~cm}$ high. When mixed flow of water and sand experiments would be carried out ,the long barrel must be filled with saturated wet sand , and then water was poured into it at the top of the sand column to form a column of water whichwas controlled in threeheight levels of $10 \mathrm{~cm}, 20 \mathrm{~cm}, 30 \mathrm{~cm}$;

ii) Camera system was ready. The plastic baffle was quickly pulled out and the inrush of sand began. At the same time, the experimental video was recorded, the experimental phenomenon was observed and a simple description was made;

iii) After the inrush of sand, the video was stopped recording. The whole experiments were conducted by changing the water content, the sand diameter, and glass bead size.

iv) After all the experiments were finished, the videos werearranged and marked;

v)The sand level readings were read every 2 seconds in dry sand experiments from the video files. And in the mixed flow of water and sand experiments, the sand level readings and the water level readings were read every 1 or 2 or 4 seconds according to the velocity of the sand inrush every experiment (Figure 2).Data was recorded in the table.

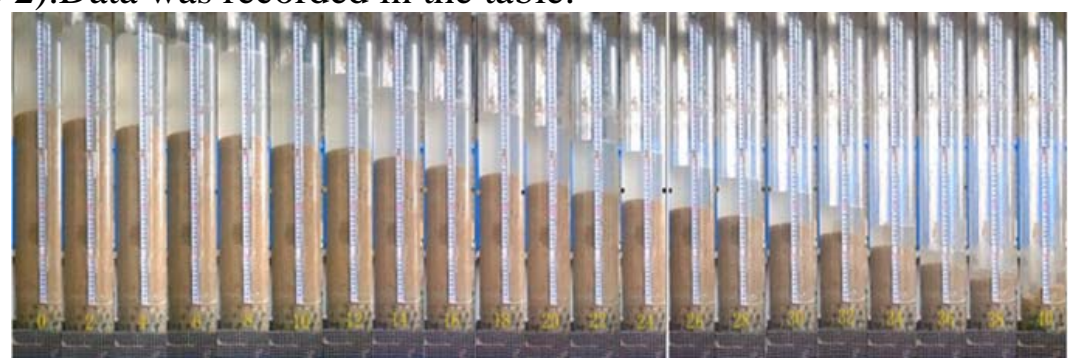

Figure 2.The phenomenon of sand level and water level changing with time

\section{Experimental Phenomenon and Result Analysis}

Experimental Phenomenon.During the dry sand experiment,in the combination of coarse sand and glass beads of the minimum diameter, a small amount of sand burstand thenoccurred block. However,in other combinations, all the sand burst and flew down completely at different velocities.

During the wet sand experiment, there was no phenomenon of sand inrush. Only when the water content got high enough, there occurred a phenomenon of dribbling water.

During the mixed flow of water sand flow experiment,water inrush and sand inrush occurred in different degree.The specific phenomenon could be divided into three:

i) The water level and the sand level fell at the same time, and the water level fellmore rapidly. Finally,when the water level was lower than that of sand, sand level fell very slowly and stopped right away.

ii) The water level and the sand level fell at the same time, and the water level fell more rapidly. But water was enough and the water column pressured sand column to fell continually. Then the sand column fell down to a certain degree, was no longer able to withstand the pressure of the overlying water column and was washed over the simulate broken zone by water column instantaneous. Finally, there is no residual sand or a little sand left on the side wall of the long barrel due to viscosity.

iii) The water level and the sand level both fell very slowly, and their velocities were almost the same. Finally, the water level and the sand level didn't change, i.e. sand inrush and water inrush stopped and the water column was separated by the sand column. This phenomenon occurred only in the experimental group with fine sand and the smallest glass beads, which formed a dense water-resisting layer so as to block the water column on the sand level. At the same time, channels formed by the broken zone were also smaller. Although water column was still on the sand column ,the sand inrush stopped.

Experimental Result Analysis.To facilitate recording and study, the factor of sand particle size was marked as A, then the sand could be denoted as A1, A2, A3 from large to small in size; the factor of glass beads size was marked as B, then the glass beads could be denoted as B 1,B2, B3, B1 from large to small in size; the factor of water column was marked as $C$, then the water columns could be 
denoted as C1, C2, C3 from high to short.

The data in the record table were analyzed. Taking time as X-axis ,sand level/water level readings as y-axis, displacement-time scatter diagrams of sand level and water level were drew with OriginPro8, and sand level-time curves were fitted by functions. Then, the influence degree of factors was compared and analyzed.

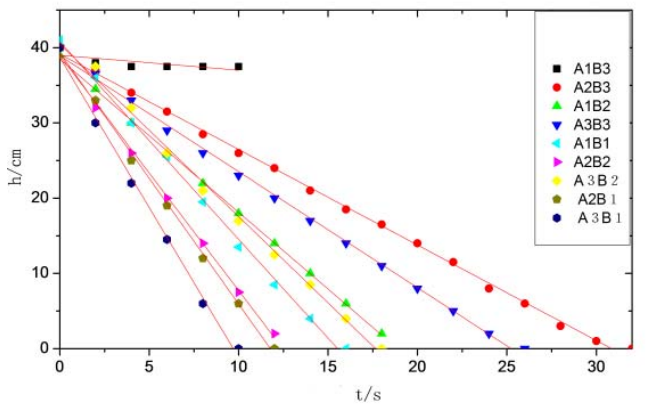

Figure 3. Dry sand displacement-time curve

During the dry sand experiment,sand level - time curves of dry sand experiments were fitted by linear function, and almost all the fitted relation coefficients were larger than 0.99 except the combination of coarse sand and the smallest glass beads experiment( Figure 3 ).It could be known that the velocity of dry sand inrush was uniform and irrelevant to the height of the sand column.

During the mixed flow of water sand flow experiment,The experimental curves of each combination of sand and glass beads with three water column gradients were drawn in a graph(Figure 4), so there were 9 graphs. Then, the curves were fitted by linear function, polynomial function and exponential function. All the fitted relation coefficients were larger than 0.95. Comparing the slopes of the three curves in each graph showed that the falling velocity of the sand column increased with the increase of the initial water column height.
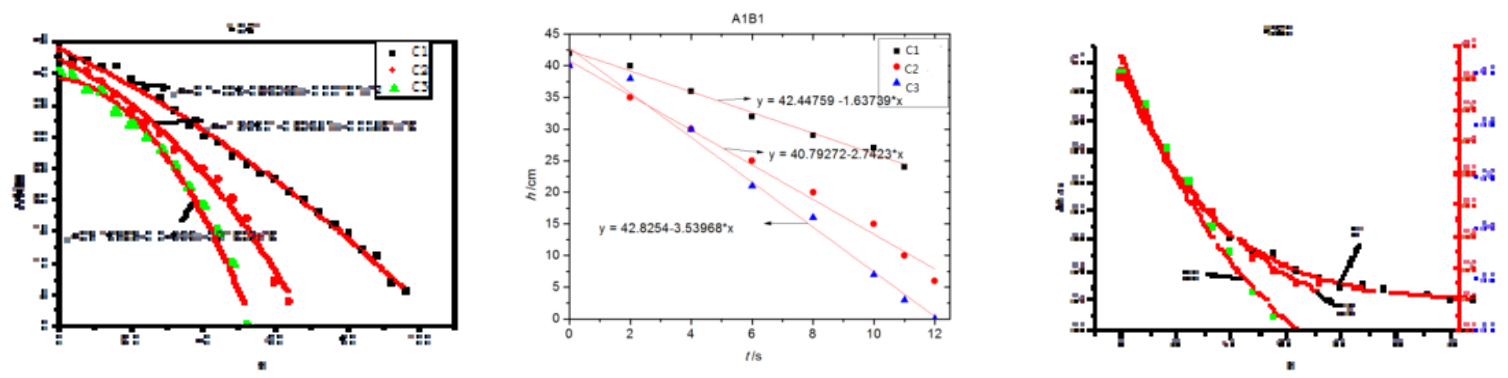

Figure 4.Parabolic, linear and exponential displacement-time curve of sand column

in the mixed flow of water and sand

Comparison of the data showed that when the mixed flow of water and sand passed through the broken rocks, the velocity of sand inrush were positively correlated with sand particle size which was contrary to the law of dry sand experiments.Calculating and comparing the data with balance-method showed that the effect order of factors on the velocity of sand inrushwas channel size $>$ sand particle size >initial water column height.

Similarly, it could be known that the amount of sand inrush was positively correlated with the initial water column height and the channel size and was negatively correlated with the sand particle size. The effect order of factors on the amount of sand inrush was the channel size> initial water column height> sand particle size. It can be seen that there was no relation between the amount of sand inrush and the velocity of sand inrush.

The mixed flow of water and sand in the process of migration could be set up as a simple mechanical model (Figure 5). In the movement, the sand body was subjected to not only a resultant force of resistance but also a resultant force of dynamic. The resultant force of resistance came from the barrier of the broken rock mass, the friction of the sand body and the rock mass, the cohesion of the sand body itself, the coupling effect of water and sand, and so on. And the resultant force of 
dynamic came from the water pressure, the gravity of the sand body itself and so on. When the resistance force was less than the dynamic force, the sand body had a downward acceleration which showed that it fell more and more quickly, and the velocity changing curve was in line with the parabola function; when the resistance force was greater than the dynamic force, the sand body had a upward acceleration which showed that the velocitywas gradually decreasing, and the velocity changing curve was in line with the exponential function; when the resistance force was equal to the dynamic force, the sand body fell at an even speed or kept static. This explained the law of the flow and block when the mixed flow of water and sand passes through in the passage.

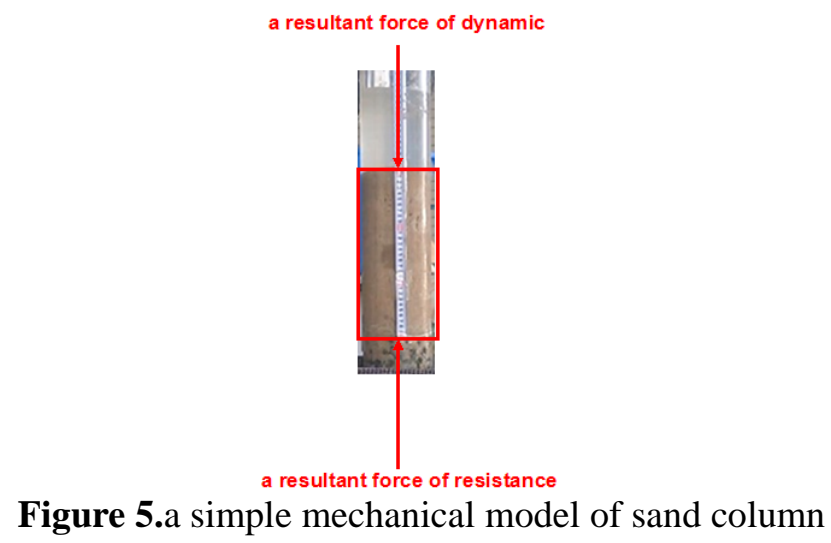

\section{Conclusion}

In this paper, a set of self-designed indoor simulated experiments for quicksand were established. The law and its characteristics of the sand flowing through the broken rock mass with different grain sizes, different water content of the sand were studied, and the main conclusions were as follows:

(1)The dry sand in the quicksand process, its sand column is uniformly falling. Obviously, the dry quicksand velocity and the ratio between the crushing channel size and the grain size is positively correlated. When the broken rock mass is small enough, the crushing channel is also very small. In this case, when the sand grain size is large enough, it would be crowded in the mouth of the channel. Thus the sand forms a smaller channel than the broken rock mass' channel, then the sand column is blocked in the broken rock mass.

(2) When the sand is containing water, and the water content is near saturation or before saturation, the cohesion force between the grains of sand increases so much, that the grains are connected to each other as a whole. At this moment, the broken rock mass is consisting of many small channels, thus the sand column cannot pass through totally. The water in this process is characterized by resistance. Thus the wet sand in the broken rock mass is relatively safe.

(3) When the water-sand mixture flows through the broken rock mass, the water level being higher than the sand is the prerequisite of quicksand, and the water plays a dynamic role. When the water drops below the sand, the sand flow becomes wet sand, so the quicksand ceases. When the water-sand mixture flows through broken rock mass, the rule of the crushing volume and the change of quicksand mixture velocity can be fitted to a linear function, a parabola and exponential functions. Water-sand mixture flowing in the migration process can be established as a mechanical model, and the mixture body in motion has a resistance joint force and a dynamic joint force. The relative magnitude of Resistance and dynamic force power determines whether water-sand mixture in the broken rock mass causes quicksand and its migration velocity curves.

(4) When the water-sand mixture flows through the broken rock mass, the average velocity of quicksand, the initial water column height, the size of the channel are correlated positively. They also have the positive correlation with sand grain size (this is the opposite to the dry sand). When the water-sand mixture flows through the broken rock mass, the sand channel size affects the quicksand velocity mostly, followed by sand grain size, and the initial water column height on the quicksand velocity has a minimal impact. 
(5)When the water-sand mixture flows through the broken rock mass, the block degree of quicksand has a negative correlation-ship with the initial water column height and quicksand channel size, and it has a positive correlation-ship with sand grain size. These three factors on the formation of blocking effect are on this order: quicksand channel size (maximum), the height of the water column, sand sample size (minimum). The quicksand amount and its velocity are not necessarily associated.

Thus, the initial water column on the sand surface is a prerequisite for the quicksand on the broken rock mass, but when the quicksand occurs, the biggest factor on the quicksand velocity and its amount, is the quicksand channel size. Therefore, in the engineering geological models, our first precondition for limiting quicksand is to reduce the water level, then to plug quicksand channels in a timely manner to prevent quicksand.

\section{Reference:}

[1] YANGWeifeng, SUI Wanghua, JI Yubing, ZHAO Guorong. Experimental research on the movement process of mixed water and sand flow across overburden fissures in thin bedrock induced by mining In Chinese[J]. Journalof China Coal Society. 37(1): 141-146, 2012

[2] YANGWeifeng. Overburden failure in thin bedrock and characteristics of mixed water and sand flow induced by mining In Chinese[D].Xuzhou:China University of Mining and Technology.2009

[3]SUIWanghua, CAI Guangtao, DONG Qinghong. Experimental research on critical percolation gradient of quicksand across overburden fissures due to coal mining near unconsolidated soil layers In Chinese[J].Chinese Journal of Rock Mechanics and Engineering.26(10): 2086-2091,2007

[4] WUYongping, LU Mingshi.Analysis of sand inrush generation condition in coal mining of shallow coal seam In Chinese[J]. Mine pressure and roof management.2004(3): 57-61.2004

[5]LUMingshi.Research on the control mechanism of water inrush and sand inrush in shallow seam[D]. Xi'an:Xi'an University of Science and Technology.2004

[6]LIANGYankun.Experimental simulation for the flowcharacter and quantity of quicksand basedon granular flow theoryIn Chinese[D].Xuzhou:China University of Mining and Technology.2012 